\title{
A New Theoretical Model of a Carbon Nanotube Strain Sensor*
}

\author{
QIU Wei(仇巍 $)^{1 * *}$, KANG Yi-Lan(元一阑 $)^{1}$, LEI Zhen-Kun(雷振坤 $)^{2}$, QIN Qing-Hua(秦庆华 $)^{3}$, LI Qiu(李秋 $)^{1}$ \\ ${ }^{1}$ Department of Mechanics, Tianjin University, Tianjin 300072 \\ ${ }^{2}$ Department of Engineering Mechanics, Dalian University of Technology, Dalian 116024 \\ ${ }^{3}$ Department of Engineering, Australian National University, Canberra ACT 2601, Australia
}

(Received 27 March 2009)

\begin{abstract}
Carbon nanotubes (CNTs) are potential strain sensors due to their excellent mechanical and spectral properties. A new theoretical model of a CNT strain sensor is obtained by applying the polarized Raman properties of CNTs, which calculates the synthetic contributions of Raman spectra from the CNTs in random directions. By using this theoretical model, the analytic relationship between planar strain components and the Raman shift increment of uniformly dispersed CNTs is obtained, which is applicable for accurately characterizing the strain in random directions on the surface of a measured microsystem.
\end{abstract}

PACS: 07.10.Pz, 81.70.Fy, 81.05.Tp

The manufacturing technologies ${ }^{[1]}$ property characterizing approaches, ${ }^{[2]}$ and applications as different sensors ${ }^{[3-10]}$ of carbon nanotubes (CNTs) have made remarkable progresses in recent years. It is well known that a CNT has outstanding mechanical characteristics, ${ }^{[11,12]}$ its $G^{\prime}$ band Raman shift is very sensitive to axial deformation, ${ }^{[13-15]}$ and its polarized resonant Raman exhibits the so-called antenna effect. ${ }^{[16-18]}$ All these properties make carbon nanotubes potentially robust, nondestructive and wireless sensors applicable for the measurement of strain components, which have proved elusive in practice but are expected to have an important role in future devices based on microsystems. ${ }^{[19]}$ Wagner et al. ${ }^{[20-22]}$ presented the idea of the CNT strain sensor with Raman spectroscopy and introduced two methods ${ }^{[21-23]}$ to realize that idea: non-polarized Raman for oriented CNTs and polarized Raman for stochastic CNTs. The former is applicable for measuring strain in a unique direction only, whereas the purpose of the latter is to detect the normal strain in any direction by observing the Raman shift increment $\Delta \Omega^{(\varphi)}$ obtained in a given polarizing direction $\varphi$ and regarding $\Delta \Omega^{(\varphi)}$ as directly proportional to the normal strain in the same direction,

$$
\Delta \Omega^{(\varphi)}=\Psi_{\mathrm{PM}} \cdot \varepsilon_{\mathrm{PM}}(\varphi),
$$

where $\Psi_{\mathrm{PM}}$ is the so-called strain-Raman shift coefficient achieved by calibration, and $\varepsilon_{\mathrm{PM}}(\varphi)$ is the measured value of the normal strain in the $\varphi$ direction. However, this is an approximate method and can be used in some fixed conditions for strain testing. Moreover, there is still a lack of theoretical study for accurately modeling a CNT sensor of strain component measurement through Raman.

In this Letter, we present a theoretical analysis of CNT strain sensors. The contribution of deformed
CNTs in any direction to the Raman spectrum is discussed by analyzing CNT polarized Raman properties and then using the proposed theoretical model, which yields the analytic relationship between the planar strain components and the Raman shift increment of uniformly dispersed CNTs.
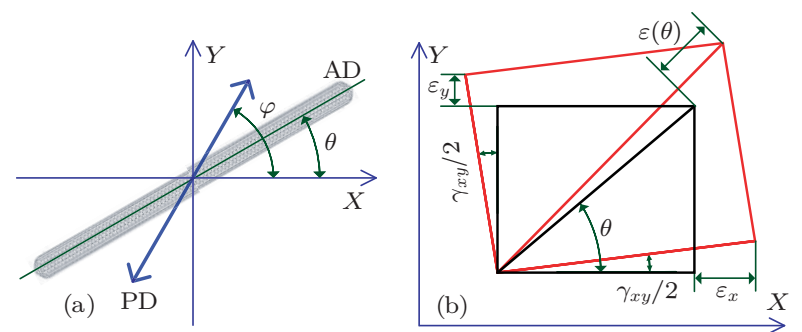

Fig. 1. Diagrammatic sketches of (a) a single CNT fixed on and deformed by a plane in the $X-Y$ Cartesian coordinate system; (b) three planar strain components.

The theoretical model of the CNT strain sensor is developed as follows. With reference to a CNT fixed on a deformable plane in the $X-Y$ Cartesian coordinate system shown in Fig. 1(a), $\theta$ is the CNT axis direction $(\mathrm{AD})$ and $\varphi$ is the polarizing direction $(\mathrm{PD})$ of the Raman spectroscope in the backscattering geometry. When the plane deforms little, the axial strain of the nanotube $\varepsilon(\theta)$ is similar to the normal strain of its location on the plane in the $\theta$ direction, which can be expressed by the three planar strain components $\varepsilon_{X}, \varepsilon_{Y}$ and $\gamma_{X Y}$ (as shown in Fig. 1(b)). Meanwhile, it has been proved ${ }^{[15]}$ that CNT axial strain $\varepsilon(\theta)$ is directly proportional to the increment of $G^{\prime}$ band Raman shift in its Raman spectrum, denoted as $\Delta \boldsymbol{\omega}_{(\theta)}$. Therefore,

$$
\begin{aligned}
\Delta \omega_{(\theta)}= & \Psi_{\text {Sensor }} \cdot \varepsilon(\theta)=\Psi_{\text {Sensor }} \cdot\left(\varepsilon_{X} \cos ^{2} \theta\right. \\
& \left.+\varepsilon_{Y} \sin ^{2} \theta-\gamma_{X Y} \cos \theta \sin \theta\right) .
\end{aligned}
$$

\footnotetext{
* Supported by the National Natural Science Foundation of China under Grant Nos 10732080 and 10502014.

**Email: qiuwei@tju.edu.cn

(C) 2009 Chinese Physical Society and IOP Publishing Ltd
} 
Equation (2) represents the analytic relationship between the planar strain components and the Raman shift increment of a single CNT in the $\theta$ direction, where $\Psi_{\text {Sensor }}$ is defined as the strain-Raman shift coefficient of the CNT strain sensor.

It is well known that the spectrum curve of the CNT $G^{\prime}$ band $I_{(\theta)}^{(\varphi)}(x)$ can be described by a Lorentz or Gauss function. Taking the Lorentz function as an example, we have

$$
\begin{aligned}
I_{(\theta)}^{(\varphi)}(x) & =\frac{\boldsymbol{R}}{\left(\frac{x-\omega_{(\theta)}}{\lambda}\right)^{2}+1^{2}} \\
& =A_{S C} \cdot \frac{1}{\pi} \frac{\lambda}{\left(x-\boldsymbol{\omega}_{(\theta)}\right)^{2}+\lambda^{2}},
\end{aligned}
$$

where $R$ is the peak height of the spectrum curve, corresponding to the scattering intensity of the $G^{\prime}$ band. Actually, $R$ is a function of the angle between $\mathrm{AD}$ and PD, $(\theta-\varphi)$, owing to the polarized Raman properties of CNTs. ${ }^{[17,24]} \boldsymbol{\omega}_{(\theta)}$ is the peak location, corresponding to the Raman shift, whose increment is sensitive to the CNT deformation in AD as Eq. (2) shows; $\lambda$ is the half-width at half-maximum (HWHM), which is treated as a constant in this Letter since it is insensitive to both the axial deformation and the polarized direction, $A_{S C}$ is the area between the curve and $X$ axis, $A_{S C}=\pi \lambda R$. According to Eq. (3), the normalized form of $I_{(\theta)}^{(\varphi)}(x)$, denoted as $T_{(\theta)}^{(\varphi)}(x)$, conforms to the Cauchy distribution,

$$
\begin{aligned}
& T_{(\theta)}^{(\varphi)}(x)=\frac{I_{(\theta)}^{(\varphi)}(x)}{\boldsymbol{A}_{S C}} \\
= & \frac{1}{\pi} \frac{\lambda}{\left(x-\boldsymbol{\omega}_{(\theta)}\right)^{2}+\lambda^{2}} \sim \mathcal{C}\left(\boldsymbol{\omega}_{(\theta)}, \lambda\right) .
\end{aligned}
$$

Consider a solid body to be measured (see Fig. 2(a)). Some CNTs are affixed onto the surface of the measured body horizontally, uniformly and stochastically (as shown in Fig. 2(b)), and may deform together with the body. The spectrum information of the Raman test on these uniformly dispersed CNTs, denoted as $I_{A C}^{(\varphi)}(x)$, is the summation of the scattering data from all the single CNTs inside the sampling area. From the viewpoint of statistics, since they are distributed uniformly and stochastically, the CNTs within a random infinitesimal angle range $d \theta$ on the $X-Y$ surface make up $\pi^{-1} d \theta$ of the total. Hence,

$$
\begin{gathered}
I_{A C}^{(\varphi)}(x)=\int_{-\pi / 2}^{\pi / 2} I_{(\theta)}^{(\varphi)}(x) \cdot \frac{1}{\pi} d \theta, \\
A_{A C}=\int_{-\pi / 2}^{\pi / 2} \boldsymbol{A}_{I C} \cdot \frac{1}{\pi} d \theta=\lambda \int_{-\pi / 2}^{\pi / 2} \boldsymbol{R} d \theta .
\end{gathered}
$$

Normalizing $I_{A C}^{(\varphi)}(x)$ into $Z^{(\varphi)}(x)$, we have

$$
Z^{(\varphi)}(x)=\frac{I_{A C}^{(\varphi)}(x)}{A_{A C}}=\int_{-\pi / 2}^{\pi / 2}\left[C_{(\theta)}^{(\varphi)} \cdot T_{(\theta)}^{(\varphi)}(x)\right]
$$

$$
\text { where } C_{(\theta)}^{(\varphi)}=\frac{\boldsymbol{R} \cdot d \theta}{\int_{-\pi / 2}^{\pi / 2} \boldsymbol{R} \cdot d \theta} .
$$

It can be seen that $Z^{(\varphi)}(x)$ can be regarded as the linear accumulation of numerous independent Cauchy distributions $T_{(\theta)}^{(\varphi)}(x) \sim \mathcal{C}\left(\omega_{(\theta)}, \lambda\right)$ with respective weight $C_{(\theta)}^{(\varphi)}$. In response to the properties of a Cauchy distribution, ${ }^{[25]} Z^{(\varphi)}(x)$ is also a Cauchy distribution and its peak location $\Omega^{(\varphi)}=\int_{-\pi / 2}^{\pi / 2}\left[C_{(\theta)}^{(\varphi)} \cdot \omega_{(\theta)}\right]$. Then, making use of Eqs. (2) and (6), the analytical relationship between the planar strain components and the Raman shift increment of uniformly dispersed CNTs is obtained,

$$
\begin{aligned}
\Delta \Omega^{(\varphi)}= & \left(\int_{-\pi / 2}^{\pi / 2} \boldsymbol{R} d \theta\right)^{-1} \int_{-\pi / 2}^{\pi / 2} \Psi_{\text {Sensor }} \cdot\left(\varepsilon_{X} \cos ^{2} \theta\right. \\
& \left.+\varepsilon_{Y} \sin ^{2} \theta-\gamma_{x y} \cos \theta \sin \theta\right) \cdot \boldsymbol{R} d \theta
\end{aligned}
$$

The above analytical relationship can be simplified by using the mathematical expression of the scattering intensity $\boldsymbol{R}$, which is determined according to the component configuration of the polarized Raman spectroscope and the scattering state of CNTs. ${ }^{[17,18,24]}$ For instance, when the polarizing directions of the incident and scattered light in the Raman spectroscope are controlled to remain constantly parallel to each other, the polarized Raman scattering of CNTs is meanwhile in a resonant state ${ }^{[26,27]}$ and exhibits the "antenna effect", $\boldsymbol{R}=\kappa \cos ^{4}(\theta-\varphi)$ and $\kappa=$ const. ${ }^{[18]}$ Hence Eq. (7) can be simplified to

$$
\begin{aligned}
\Delta \Omega^{(\varphi)}= & \frac{1}{6} \Psi_{\text {Sensor }} \cdot\left[(3+2 \cos 2 \varphi) \varepsilon_{X}+(3\right. \\
& \left.-2 \cos 2 \varphi) \varepsilon_{Y}-2 \sin 2 \varphi \cdot \gamma_{X Y}\right]
\end{aligned}
$$

It can be seen from Eq. (8) that the Raman shift increment achieved in any appointed PD is equal to the summation of three planar strain components with dissimilar trigonometric functions relative to the PD as weighted parameters. Therefore, by using the CNTs as strain sensors, we can study the strain state of the measured body by detecting several Raman shift increments of the CNTs in different PDs.

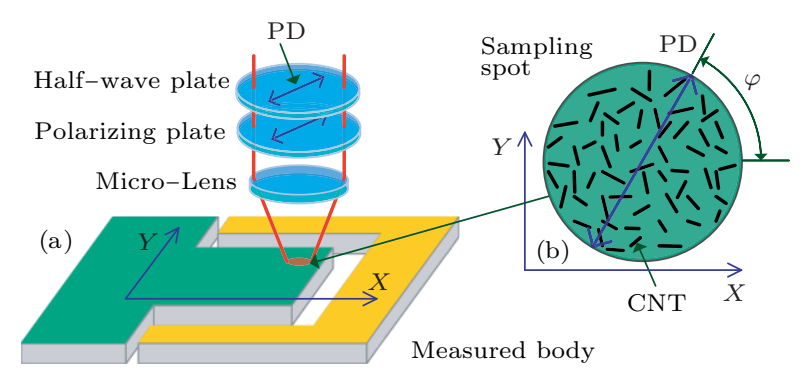

Fig. 2. Diagrammatic sketches of CNT strain sensors using polarized Raman spectroscopy. (a) The measured body and Raman system. (b) CNTs dispersed uniformly on the surface of the measured body. 
Equation (7) can be simplified to other forms under different experimental modes such as "only the PD of incident light controllable" [17] and "CNT scattering in non-resonant state". ${ }^{[24]}$ Meanwhile, Eq. (7) is also applicable when CNTs disperse non-stochastically or even when they are oriented by introducing a distributing density function $\rho(\theta)$ to replace $\pi^{-1}$ in Eq. (5). In addition, if the spectral curve of a CNT is taken as a Gauss function, the derivation also results in the same formulation.

To confirm the theoretical model in this work, a uniaxial tensile test was performed on a specimen of free-standing CNT composite film. The matrix was a DGEBA-based epoxy, and single-wall CNTs functioned by the $-\mathrm{COOH}$ group (0.5 wt.\%, TIMESNANO Ltd) were stochastically dispersed in liquid epoxy by ultrasound for $24 \mathrm{~h}$. The cured film was about $160 \mu \mathrm{m}$ thick with a Young's modulus of $2.00 \mathrm{GPa}$ and Poisson ratio 0.379 . The specimen was tested in a minitensile machine, with the loading direction parallel to the $X$ direction, using a step length of $0.04 \%$ when $\varepsilon_{X}<0.72 \%$ and $0.2 \%$ when $\varepsilon_{X} \geq 0.72 \%$ until the specimen broke. A Renishaw InVia Raman spectroscope together with a He-Ne laser source $(632.8 \mathrm{~nm}$, $2 \mathrm{~mW}$ ) were utilized and the incident beam was focused on the specimen surface with the backscattering geometry through a $50 \times$ objective lens, forming a sampling spot of about $2 \mu \mathrm{m}$ in diameter. Under each loading step, Raman spectra from the sampling spot in $0^{\circ} / 45^{\circ} / 90^{\circ} \mathrm{PDs}$ were recorded.

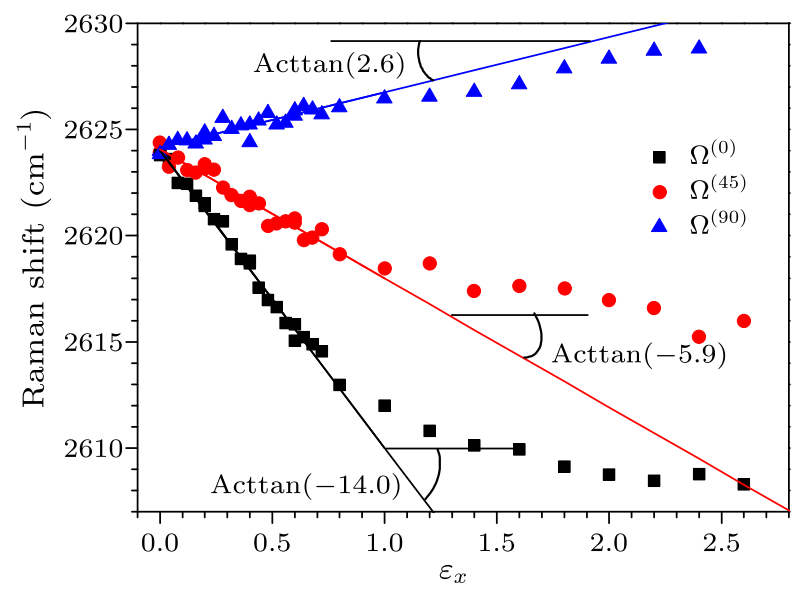

Fig. 3. Raman shifts of the test on the free-standing CNT film.

All the raw data were fitted by a Lorentz function to achieve the Raman shifts shown in Fig. 3. It can be seen that all the Raman shifts, $\Omega^{(0)}, \Omega^{(45)}$ and $\Omega^{(90)}$, start from about $2624 \mathrm{~cm}^{-1}$ and change linearly with the increase of tensile loading until $\varepsilon_{X} \geq 0.8 \%$. Their slopes in the linearity range, $\Delta \Omega^{(0)} / \varepsilon_{X}, \Delta \Omega^{(45)} / \varepsilon_{X}$ and $\Delta \Omega^{(90)} / \varepsilon_{X}$, are $-14.0,-5.9$ and 2.6 , respectively. By dividing both sides of Eq. (8) by $\varepsilon_{X}$ and then substituting the Raman shift slopes in Fig. 3 and their respective $\mathrm{PDs}$, we obtain

$$
\Psi_{\text {Sensor }}=-1815 \mathrm{~cm}^{-1}, \quad \nu=-\frac{\varepsilon_{Y}}{\varepsilon_{X}}=0.372 .
$$

From Eq. (9), it can be seen that the experimental results are consistent with the theoretical ones. In particular, the Poisson ratio obtained by means of our CNT strain sensor, 0.372 , is almost equal to its true value, 0.379 .

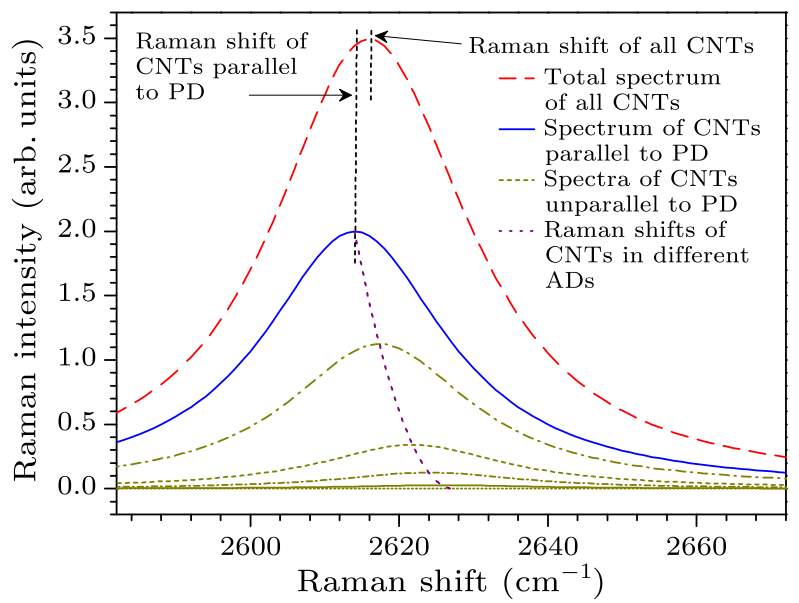

Fig. 4. Diagrammatic sketch of the contribution of CNTs with different axial directions (ADs) to the total spectrum in any appointed polarized direction (PD).

The above experiment shows that Eq. (8) can represent the planar strain state of the measured body accurately, because the theory in this study considers the synthetic contributions of Raman information from the CNTs in all the directions within the plane. In contrast, Eq. (1) indicates that only the scatterings from CNTs parallel to the PD are counted, so that the measuring result by the previous method ${ }^{[22,23]}$ is only approximate to or even far from the true value. This is because every CNT makes a contribution to the total spectrum and indeed none is negligible. Figure 4 gives a diagrammatic sketch of the contribution of CNTs with different axial directions (ADs) to the total spectrum in any appointed polarized direction (PD). It shows that the contributions of individuals to the total are not average. Even though the scattering data of CNTs parallel to the PD are dominant in the total spectrum due to the antenna effect ${ }^{[16]}$ of CNTs in polarized Raman scattering, those from CNTs not parallel to the PD exert an influence, making the Raman shift of the total spectrum from all CNTs different from that of the CNTs parallel to the PD. As shown in Fig. 4, the distinct distance between the two Raman shifts demonstrates that the Raman shift increment $\Delta \Omega^{(\varphi)}$ comprises the entire contributions of all the CNTs, and should not be in direct proportion to the normal strain in the $\varphi$ direction as Eq. (1) presumes.

Based on the above model, the CNT strain sensor may be applied by the following steps. Firstly, a 
CNT film is prepared on the surface of a microsystem by means of self-assembly, printing, pasting or some other method. $\Psi_{\text {Sensor }}$ is calibrated by means of basic experiments. Then, the Raman spectra in several different PDs are detected through polarized Raman tests, and the raw data are fitted to obtain the Raman shifts and substituted into Eq. (8) respectively, building an equation set. By solving this equation set, the values of $\varepsilon_{X}, \varepsilon_{Y}$ and $\gamma_{X Y}$ are achieved.

In conclusion, a new theory of CNT strain sensors is developed and verified by experimental example. By calculating the synthetic contributions of Raman information from the CNTs in all directions and utilizing the Raman properties of CNTs including Raman shift sensitivity to strain, polarized and resonant Raman, an analytic relationship between the planar strain components and the Raman shift of uniformly dispersed CNTs is obtained. The experimental results and discussions of their contrast demonstrate that the CNT strain sensors in this work may wirelessly and accurately characterize the strain in random directions on the surface of a microsystem.

\section{References}

[1] Dai H J 2002 Account. Chem. Res. 351035

[2] Terrones M 2003 Ann. Rev. Mater. Res. 33419

[3] Collins P G, Bradley K, Ishigami M and Zettl A 2000 Science 2871801

[4] Kong J, Chapline M G and Dai H J 2001 Adv. Mater. 13 1384

[5] Modi A, Koratkar N, Lass E, Wei B Q and Ajayan P M 2003 Nature 424171

[6] Lin Y H, Lu F, Tu Y and Ren Z F 2004 Nano Lett. 4191

[7] Wang J 2005 Electroanalysis 177

[8] Barone P W, Baik S, Heller D A and Strano M S 2005
Nature Mater. 486

[9] Wu J A, Zang J, Larade B, Guo H, Gong X G and Liu F 2004 Phys. Rev. B 69153406

[10] Wood J R and Wagner H D 2000 Appl. Phys. Lett. 76 2883

[11] Bernholc J, Brenner D, Nardelli M B, Meunier V and Roland C 2002 Ann. Rev. Mater. Res. 32347

[12] Cao J, Wang Q and Dai H J 2003 Phys. Rev. Lett. 90 157601

[13] Cooper C A, Young R J and Halsall M 2001 Composites A 32401

[14] Kao C C and Young R J 2004 Compos. Sci. Technol. 64 2291

[15] Cronin S B, Swan A K, Unlu M S, Goldberg B B, Dresselhaus M S and Tinkham M 2005 Phys. Rev. B 72035425

[16] Dresselhaus M S, Dresselhaus G, Saito R and Jorio A 2005 Phys. Rep. 40947

[17] Duesberg G S, Loa I, Burghard M, Syassen K and Roth S 2000 Phys. Rev. Lett. 855436

[18] Gommans H H, Alldredge J W, Tashiro H, Park J, Magnuson J and Rinzler A G 2000 J. Appl. Phys. 882509

[19] Hytch M, Houdellier F, Hue F and Snoeck E 2008 Nature 4531086

[20] Wood J R, Zhao Q, Frogley M D, Meurs E R, Prins A D, Peijs T, Dunstan D J and Wagner H D 2000 Phys. Rev. B 627571

[21] Zhao Q, Wood J R and Wagner H D 2001 Appl. Phys. Lett. 781748

[22] Zhao Q, Frogley M D and Wagner H D 2002 Polym. Adv. $T 13759$

[23] Frogley M D, Zhao Q and Wagner H D 2002 Phys. Rev. B 65113413

[24] Saito R, Dresselhaus G and Dresselhaus M S 1998 Physical Properties of Carbon Nanotubes (London: Imperial College)

[25] Derman C, Gleser L J and Olkin I 1973 A Guide to Probability Theory and Application (New York: Holt)

[26] Pimenta M A, Marucci A, Empedocles S A, Bawendi M G, Hanlon E B, Rao A M, Eklund P C, Smalley R E, Dresselhaus G and Dresselhaus M S 1998 Phys. Rev. B 58 R16016

[27] Jorio A, Dresselhaus G, Dresselhaus M S, Souza M, Dantas M S S, Pimenta M A, Rao A M, Saito R, Liu C and Cheng H M 2000 Phys. Rev. Lett. 852617 